describes the percentage variation in red cell size. The neutrophil and lymphocyte count can be used to calculate a ratio (NLR). Limited published work has evaluated the prognostic significance of these markers in IPF. Our aim was to assess longitudinal changes in RDW and NLR as potential prognosticators in IPF.

Methods Patients with IPF were identified from the Royal Devon and Exeter Hospital (2005-2019). Data collected: baseline characteristics, survival, PFTs and FBC $<6$ months from diagnosis and 6-18 months during follow-up. Patients with insufficient data were excluded. Blood parameters were stratified into quartiles for subsequent Kaplan-Meier survival analyses, Mann-Whitney U-test and Spearman's rank correlation.

Results 131 patients were included in analysis. Median change/month for NLR (deltaNLR) and RDW (deltaRDW) were 0.17 and 0.02 respectively, indicating minimal longitudinal variation. Anti-fibrotic treatment did not modify deltaRDW or deltaNLR. However, stratifying by median deltaRDW significantly impacted on survival (median 33 months with deltaRDW $>0.02$ vs 59 months; $\mathrm{P}=0.04)$. Median survival stratified on baseline RDW was 35 months (highest quartile) vs 47 months (1st-3rd quartiles) although this did not reach significance $(P=0.1439)$. Median survival based on follow-up RDW was 25 months (highest quartile) vs 59 months (1st-3rd quartiles; $\mathrm{P}=0.0021$ ) and this was negatively correlated with FVC $(\mathrm{P}=0.0056)$. Both baseline and follow-up NLR had significantly shorter median survival in the highest quartile ( 28 months; $\mathrm{p}<0.05$ ) compared with 47 months (baseline 1st-3rd quartile) or 59 months (followup 1 st-3rd quartile). FVC was negatively correlated to baseline NLR $(\mathrm{P}=0.0282)$.

Conclusions RDW and NLR demonstrated significant relationships with survival and correlations with FVC. Increasing RDW resulted in poorer outcomes. Although limited by the small retrospective cohort, this data indicates that readily available FBC may have utility in prognostication and progression monitoring in IPF, independent of antifibrotic treatment. RDW may be confounded by co-morbidities; further work to assess this is warranted.

\section{P145 MARGINAL SHORT TERM LUNG FUNCTION CHANGES PREDICT MORTALITY IN PATIENTS WITH FIBROTIC HYPERSENSITIVITY PNEUMONITIS}

${ }^{1,2} \mathrm{C}$ Macaluso, ${ }^{1,3} \mathrm{C}$ Boccabella, ${ }^{1} \mathrm{M}$ Kokosi, ${ }^{1} \mathrm{~V}$ Kouranos, ${ }^{1,4} \mathrm{PM}$ George, ${ }^{1,5,6} \mathrm{G}$ Margaritopoulos, ${ }^{1,4} \mathrm{PL}$ Molyneaux, ${ }^{1} \mathrm{~F}$ Chua, ${ }^{1,4,7} \mathrm{TM}$ Maher, ${ }^{1,4} \mathrm{GR}$ Jenkins, ${ }^{8} \mathrm{~S}$ Desai, ${ }^{8} \mathrm{~A}$ Devaraj, ${ }^{1,4} \mathrm{AU}$ Wells, ${ }^{1,4} \mathrm{EA}$ Renzoni, ${ }^{1,4} \mathrm{CJW}$ Stock. ${ }^{1}$ Interstitial Lung Disease Unit, Royal Brompton and Harefield Clinical Group, Guy's and St Thomas' NHS Foundation Trust, London, UK; ' ${ }^{2}$ INRCAIIRCCS, 'L.Mandic' Hospital Merate (LC) - Department of Pneumology, Merate, Italy; ${ }^{3}$ Department of Medical and Surgical Sciences, Fondazione Policlinico Universitario 'A. Gemelli' - IRCCS, University of the Sacred Heart, Rome, Italy; ${ }^{4}$ Margaret Turner Warwick Centre for Fibrosing Lung Disease, National Heart and Lung Institute, Imperial College London, London, UK; ${ }^{5}$ LLD Unit, London North West University Hospital Healthcare Trust, London, UK; ${ }^{6}$ Faculty of Biology, Medicine and Health, The University of Manchester, Manchester, UK; ${ }^{7}$ Hastings Centre for Pulmonary Research and Division of Pulmonary, Critical Care and Sleep Medicine, Keck School of Medicine, University of Southern California, Los Angeles, USA; ${ }^{8}$ Department of Radiology, Royal Brompton and Harefield Clinical Group, Guy's and St Thomas' NHS Foundation Trust, London, UK

\subsection{6/thorax-2021-BTSabstracts.254}

Background and Objective A proportion of patients with fibrotic hypersensitivity pneumonitis (fHP) follow a progressive disease course despite immunosuppressive treatment. Little is known about predictors of mortality in fHP. We aimed to investigate the impact of marginal, short-term lung function changes in fHP on mortality.

Methods Baseline demographics were recorded for 145 consecutive patients with a Multi-Disciplinary Team diagnosis of fHP, as well as baseline and one year follow-up lung function, baseline echocardiographic findings, bronchoalveolar lavage (BAL) cellularity, and all-cause mortality. Marginal changes in FVC $\geq 5 \%$ and DLCO $\geq 10 \%$ at one year were calculated. Cox proportional hazards analysis was performed to test for associations with mortality.

Results Baseline lung function severity (FVC, DLCO, and composite physiological index (CPI)), age, and PASP $\geq 40 \mathrm{~mm} \mathrm{Hg}$ on echocardiogram were associated with early mortality, while BAL lymphocytosis was associated with improved survival. A marginal decline at one year in FVC $\geq 5 \%$ (HR: 3.10 , 95\% CI: 2.00-4.81, p<0.001) and DLCO $\geq 10 \%$ (HR: $2.80(95 \%$ CI: 1.78-4.42), $\mathrm{p}<0.001)$ were associated with markedly
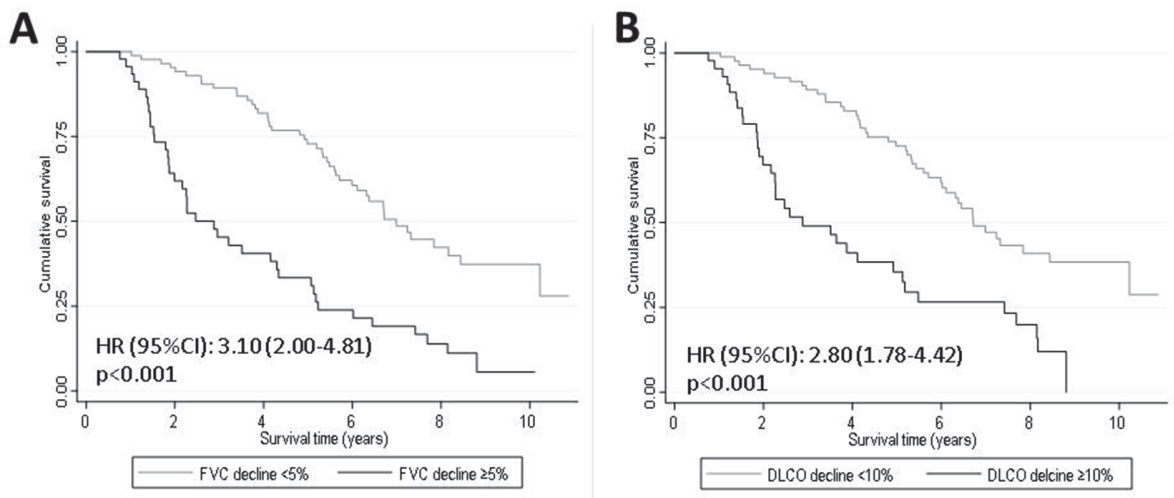

Kaplan-Meier survival analysis grouped by decline at one year in (A) FVC $\geq 5 \%$ and $(B)$ DLCO $\geq 10$.

Abstract P145 Figure 1 Survival according to decline in lung function at one year 
reduced survival on univariable analysis. Both of these associations remained significant on multivariable analysis correcting for demographic variables, disease severity (as each of: CPI, FVC, and DLCO), and treatment. The association of both measures of decline with early mortality were also maintained when, in separate models, PASP $\geq 40 \mathrm{~mm} \mathrm{Hg}$ on echocardiography, and BAL lymphocytosis thresholds of $20 \%, 30 \%$ and $40 \%$, were included in addition to demographic variables, disease severity, and treatment.

Conclusions A marginal worsening in FVC of $\geq 5 \%$ and in DLCO of $\geq 10 \%$ at one year are predictive of markedly reduced survival in $\mathrm{fHP}$.

\section{P146 PREDICTORS OF ADVERSE OUTCOME IN SARCOIDOSIS COMPLICATED BY PULMONARY ASPERGILLOSIS}

${ }^{1} \mathrm{~L}$ Nwankwo, ${ }^{2} \mathrm{~J}$ Periselneris, 'D Gilmartin, ${ }^{1} \mathrm{~S}$ Desai, ${ }^{1} \mathrm{~A}$ Shah, ${ }^{1} \mathrm{~V}$ Kouranos, ${ }^{1} \mathrm{AU}$ Wells, ${ }^{1}$ E Renzoni, ${ }^{1} \mathrm{PL}$ Molyneaux, ${ }^{1} \mathrm{P}$ George, ${ }^{1} \mathrm{M}$ Kokosi, ${ }^{1} \mathrm{~A}$ Devaraj, ${ }^{1} \mathrm{D}$ Armstrong-James, ${ }^{1}$ F Chua. 'Royal Brompton Hospital, Guy's and St. Thomas' NHS Foundation Trust, London, UK; ${ }^{2}$ Kings College Hospital, London, UK

\subsection{6/thorax-2021-BTSabstracts.255}

Introduction Aspergillosis complicating pulmonary sarcoidosis is associated with high mortality. The specific prognostic impact of fibrocavitary sarcoid disease, however, remains poorly understood. A better understanding of the factors that determine adverse outcomes in such patients may improve the management of both the underlying sarcoid disease and associated secondary fungal infection.

Methods Cases of pulmonary sarcoidosis with elevated Aspergillus IgG (>40 mgA/L) presenting between January 2009 and March 2021 were retrospectively identified. Controls (sarcoidosis with normal Aspergillus IgG titre) were case-matched by baseline $\%$-predicted gas transfer factor (TLco; $\pm 5 \%$ variance). Computed tomography (CT), baseline lung function and survival data were analysed.

Results Among 180 cases (high Asp IgG) and 229 controls (normal Asp IgG), no inter-group difference was evident in the median age at presentation [48 (IQR 40-58) vs 50 (IQR 42-59)] or gender (proportion female: $45.5 \%$ vs $51.1 \%$ ). Amongst the cases, 81/180 (45\%) had fibrocavitary changes, compared with $14 / 229(6.1 \%)$ of the controls $(\mathrm{P}<0.001)$. Radiologically-evident aspergilloma was present in $79 \%$ (64/ 81) of the cases with fibrocavitary sarcoidosis. These cases also had poorer lung function compared to non-fibrocavitary disease: mean $\%$-predicted forced vital capacity/FVC $71.3 \%$ vs

\section{Survival proportions}

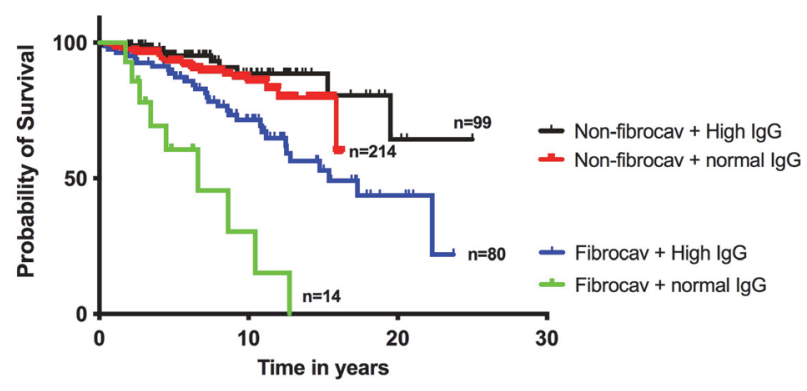

Comparison of survival curves by log-rank, $p$ value $=<0.0001$

Abstract P145 Figure 1 Survival proportions
91.4\% $(\mathrm{P}<0.0001)$, and in the controls: $69.9 \%$ vs $89.1 \%$ $(\mathrm{P}<0.01)$. A similar trend was observed in $\%$-predicted TLco amongst the fibrocavitary cases: $45.8 \%$ vs $64.2 \%$ non-fibrocavitary $(\mathrm{P}<0.0001)$ and in the fibrocavitary controls: $39.7 \%$ vs $61.8 \%$ non-fibrocavitary $(\mathrm{P}<0.0001)$. Comparing only those with fibrocavitation, neither the $\%$-predicted FVC $(71.3 \%$ vs $69.9 \%$ predicted; $\mathrm{P}=0.82)$ nor $\%$-predicted TLco $(45.7 \%$ and $39.7 \%$ predicted; $\mathrm{P}=0.17$ ) differed between cases and controls. Evidence of fibrocavitary destruction was associated with higher overall mortality (37\% vs $9.1 \%$ in the non-fibrocavitary subgroup; <0.0001) and longest median survival (graph).

Conclusions Fibrocavitary sarcoidosis is associated with worse lung function and poorer median survival. In this group, elevated Aspergillus IgG highlights a greater incidence of aspergilloma.

Implications Fibrotic transformation of pulmonary sarcoidosis heightens symptom burden, predisposes to chronic Aspergillus infection and is prognostically important particularly when there is supervening fibrocavitary lung destruction. Sensitive stratification of such patients for long-term outcome may help identify particular individuals for earlier and more focused therapeutic intervention.

\section{P147 LONG-TERM PULMONARY FUNCTION AND MORTALITY OUTCOMES IN IDIOPATHIC PULMONARY FIBROSIS PATIENTS TREATED WITH ANTIFIBROTICS}

J Barnes, E Harris, L Matos, K Harding, M Thillai. Royal Papworth Hospital NHS Foundation Trust, Cambridge, UK

\subsection{6/thorax-2021-BTSabstracts.256}

Background Idiopathic Pulmonary Fibrosis (IPF) is a progressive scarring lung disease. The antifibrotics pirfenidone and nintedanib are approved for IPF patients with a forced vital capacity $\%$ predicted (FVC\%) between 50-80\%, and either drug may be prescribed in the first instance. Both drugs reduce mortality risk, and disease progression as assessed by FVC $\%$ and transfer factor for carbon monoxide $\%$ predicted $\left(\mathrm{TL}_{\mathrm{CO}} \%\right)$ over 12-months. The effectiveness of antifibrotics beyond 12-months is less established, furthermore there is limited real world data comparing pulmonary function and mortality between both drugs.

Aims To assess the effectiveness of antifibrotics on $\mathrm{FVC} \%$ and $\mathrm{TL}_{\mathrm{CO}} \%$ over 24-months. To identify if the current clinical rationale of offering either antifibrotic from the outset is appropriate through investigating $\mathrm{FVC} \%, \mathrm{TL}_{\mathrm{CO}} \%$ and mortality outcomes in IPF patients receiving pirfenidone or nintedanib.

Methods We carried out a retrospective analysis of IPF patients with an FVC\% between 50-80\% who commenced antifibrotic treatment between May 2012 and October 2019 at Royal Papworth Hospital (Cambridge, UK). Separate random coefficient regression models were used to assess $\mathrm{FVC} \%$ and $\mathrm{TL}_{\mathrm{CO}} \%$ at $0,6,12,18$ and 24 -months. A Cox proportional hazards model was used to assess hazard ratio (HR) and 95\% confidence interval (CI) for all-cause mortality in antifibrotic treated patients.

Results 194 patients were identified from our dataset. FVC $\%$ remained stable between 0 and 12 -months $(\mathrm{p}=0.330)$ but declined between 12 and 24-months $(\mathrm{p}<0.001)$. $\mathrm{TL}_{\mathrm{CO}} \%$ decreased between 0 and 12-months $(p=0.003)$ and between 12 and 24-months $(\mathrm{p}<0.001)$. Over 24 -months, FVC\% was 